\title{
CORRECTION
}

Check for updates

Cite this: J. Mater. Chem. B, 2020, 8, 9813

DOI: 10.1039/d0tb90177f

rsc.li/materials-b

\section{Correction: Aqueous surface gels as low friction interfaces to mitigate implant-associated inflammation}

\author{
Allison L. Chau, ${ }^{a}$ Jonah Rosas, ${ }^{b}$ George D. Degen, ${ }^{c}$ Lisa K. Månsson, ${ }^{d}$ \\ Jonathan Chen, ${ }^{c}$ Eric Valois ${ }^{e}$ and Angela A. Pitenis*a
}

Correction for 'Aqueous surface gels as low friction interfaces to mitigate implant-associated inflammation' by Allison L. Chau et al., J. Mater. Chem. B, 2020, 8, 6782-6791, DOI: 10.1039/D0TB00582G.

The funding information provided for the original article was incorrect. The acknowledgements section should read:

This work was supported by the National Science Foundation (NSF) Materials Research Science and Engineering Center (MRSEC) at UC Santa Barbara through DMR-1720256 (IRG-3). The use of the shared facilities of the MRSEC is gratefully acknowledged; the UCSB MRSEC is a member of the Materials Research Facilities Network (www.mrfn.org). The authors are grateful for a California NanoSystems Institute (CNSI) Challenge Grant. J. R. was supported by the Bill and Melinda Gates Foundation. G. D. D. was supported by the National Science Foundation Graduate Research Fellowship Program under Grant No. 1650114.

The Royal Society of Chemistry apologises for these errors and any consequent inconvenience to authors and readers.

\footnotetext{
${ }^{a}$ Materials Department University of California, Santa Barbara, CA 93106, USA. E-mail: apitenis@ucsb.edu

${ }^{b}$ Biomolecular Science and Engineering Department University of California, Santa Barbara, CA 93106, USA

${ }^{c}$ Department of Chemical Engineering, University of California, Santa Barbara, Santa Barbara, CA 93106, USA

${ }^{d}$ Department of Physics Chalmers, University of Technology, 41258 Gothenburg, Sweden

${ }^{e}$ Department of Molecular, Cellular, and Developmental Biology, University of California, Santa Barbara, CA 93106, USA
} 